\title{
Space-Time Ergodic Properties of Systems of Infinitely Many Independent Particles
}

\author{
Sheldon Goldstein \\ Institute for Advanced Study, Princeton, New Jersey, USA
}

Received June 25, 1974

\begin{abstract}
We investigate the ergodic properties of the equilibrium states of systems of infinitely many particles with respect to the group generated by space translations and time evolution. The particles are assumed to move independently in a periodic external field. We show that insofar as "good thermodynamic behavior" is concerned these properties provide much sharper discrimination than the ergodic properties of the time evolution alone. In particular, we show that though the infinite ideal gas is mixing in the space-time framework, it has vanishing space-time entropy and fails to be a space-time $K$-system. On the other hand, if the particles interact with fixed convex scatterers (the Lorentz gas) the system forms a space-time $K$-system. Also, the space-time entropy of a system of the type we consider is shown to equal its time entropy per unit volume.
\end{abstract}

\section{Introduction}

One of the outstanding problems of statistical mechanics is to account for the fact that isolated macroscopic systems approach equilibrium. One expects, of course, that this approach occurs, not because of any peculiarities of actual physical systems, but because of certain general structural features relating to the nature of the interactions and the large size $\left(\sim 10^{26}\right.$ molecules) of macroscopic systems. Since thermodynamic behavior becomes exact only in the infinite volume limit, we expect that a precise mathematical formulation and justification of this behavior may be possible only for systems consisting of infinitely many particles.

We therefore want to find a mathematical framework in terms of which the problem of thermodynamic behavior can, hopefully, be understood. Typically, ergodic theory has provided such a framework; indeed, the time evolution of a finite system is mixing, an ergodic property stronger than ergodicity, in just the case where "reasonable" nonequilibrium states (i.e., absolutely continuous measures on the energy surface of the phase space) approach (weakly) the equilibrium state (microcanonical ensemble). We expect, however, that realistic systems may exhibit thermodynamic behavior without being strictly mixing; rather, large systems may be approximately mixing, in the sense that the

$\star$ Research supported in part by the National Science Foundation Grant No. GP-16147 A No. 1. 
infinite systems to which they "converge" (their infinite volume limits) exactly satisfy some useful property, analagous to mixing for finite systems, which implies that the system behave appropriately.

One is therefore led to the investigation of the ergodic properties of the time evolution of infinite classical systems [1-5] — and to the discovery that the time evolution of the infinite ideal gas has the strongest possible ergodic properties: it forms a Bernoulli flow. This implies that the system is mixing, so that states which are absolutely continuous with respect to the equilibrium state (i.e., local perturbations of the equilibrium state) approach equilibrium. We know, of course, that states of the ideal gas representing global perturbations of the equilibrium state need not approach equilibrium (e.g., a non Maxwellian velocity distribution will not become Maxwellian) and, that mixing says nothing about these (non-absolutely continuous) states. The dissipation of disturbances which occurs in the ideal gas is of a purely nonlocal nature - the local disturbances stream off to infinity where they are no longer visible.

Since an infinite system may possess such strong ergodic properties as the Bernoulli $(B)$ property without exhibiting good thermodynamic behavior, the traditional ergodic theoretic concepts (ergodicity, mixing, $K$-system, Bernoulli), all of which are implied by the $B$ property, cannot adequately account for such behavior. In fact, the situation may be somewhat worse. It appears at least plausible that infinite systems of interacting particles which exhibit good thermodynamic behavior also form $B$-flows $[2,6]$. If this were the case, a theorem of Ornstein (see [7]), implying that $B$-flows of infinite entropy are isomorphic, would imply that these systems are indistinguishable from the infinite ideal gas insofar as the measure theoretic structure of their time evolutions is concerned ${ }^{1}$. Thus, as well as new (ergodic theoretic) concepts, we need an expanded framework to support these concepts.

Fortunately, there is a rather prominent additional element of structure common to infinite systems of interest in statistical mechanics: invariance under space translations. The dynamics as well as the equilibrium states of infinite systems of particles are normally required to be translation invariant. Thus, the measure spaces of these systems possess, in a natural way, a larger invariance group than previously considered [9]: the abelian group generated by space and time translations. The ergodic properties of the equilibrium states of these systems under space translations have already been investigated (see [9]). It thus appears natural to extend our abstract framework by replacing the flow $\left\{T_{t}\right\}$ in the quadruple $\left(X, \Sigma, \mu,\left\{T_{t}\right\}\right)$ (see [10]) by the larger abelian group $\mathscr{I}$ generated by space translations and time evolution (the

1 The time evolutions of infinite systems of particles typically have infinite $(K-S)$ entropy [8], as do their space translations. 
space-time group). Generalizations of the ergodic theoretic concepts and results for a group generated by a single automorphism (or flow) to a group generated by several commuting automorphisms (or flows) have been obtained ${ }^{2}[11,12]$. We shall show that the ergodic properties of infinite systems relative to this framework provide a much sharper tool of investigation than the ergodic properties with respect to space translations or time evolution separately.

The extension of our framework to the larger group $\mathscr{I}$ has as an immediate consequence that the implications of Ornstein's theorem no longer afford us significant difficulty; though Ornstein's theorem presumably extends to a generalized dynamical system $[12][(X, \Sigma, \mu, \mathscr{I})$, $\mathscr{I}$ a group generated by several commuting automorphisms] it should be much more difficult for infinite systems to be Bernoulli under the spacetime group ${ }^{3}$.

It seems reasonable to expect that good mixing properties under the space-time group might require more than a "purely nonlocal dissipation of disturbances". We will see that the inclusion of space translations in the automorphism subgroup allows us to control effects due to the infinite extension of our systems; we shall see that though possessing infinite time evolution entropies, the infinite systems which we will consider have physically significant space-time entropies.

\section{Poisson Systems ${ }^{4}$}

Since the systems which we investigate are of noninteracting particles, they can be conveniently represented as Poisson systems: Let $(X, \Sigma, \mu)$ be a totally $\sigma$-finite nonatomic measure space ${ }^{5}$. Denote by $\bar{X}$ the set of countable subsets of $X$, and for $A \in \Sigma$ let $N_{A}: \bar{X} \rightarrow \mathbb{R}$ be given by

$$
N_{A}(\bar{x})=\#(A \cap \bar{x}),
$$

where $\bar{x} \in \bar{X}$, and "\#" stands for "the cardinality of". Let $\bar{\Sigma}$ be the smallest $\sigma$-algebra for which all of the $N_{A}$ are measurable, and denote by $\bar{\mu}$ the probability measure on $(\bar{X}, \bar{\Sigma})$ representing a Poisson distribution of points in $X$ with density given by $\mu$, i.e., the measure for which the functions $N_{A}$ have distribution given by

$$
\bar{\mu}\left\{\bar{x} \in \bar{X} \mid N_{A}(\bar{x})=m\right\}=\exp (-\mu(A)) \mu(A)^{m} / m !^{6} .
$$

${ }^{2}$ We shall review some of them in the third section of the present article.

${ }^{3}$ See Section 3.

${ }^{4}$ We shall assume that all measure spaces to which we refer are Lebesgue spaces (see [13] and [14], pp. 34 and 106).

${ }^{5}$ We use the terminology of Halmos [15].

${ }^{6}$ This condition (for all $A \in \Sigma$ ) implies the independence of disjoint regions $\subset X$ (see [16]) 
For $T$ an automorphism of $(X, \Sigma, \mu)$ define an automorphism $\bar{T}$ of $(\bar{X}, \bar{\Sigma}, \bar{\mu})$ by

$$
\bar{T} \bar{x}=T \bar{x}
$$

for $X \supset \bar{x} \in \bar{X}$. We will call $(\bar{X}, \bar{\Sigma}, \bar{\mu}, \bar{T})$ the Poisson system built over $(X, \Sigma, \mu, T)$.

We indicate a simple way (a Bernoulli construction) in which a Poisson system may be shown to be a Bernoulli shift. Recall that $(\bar{X}, \bar{\Sigma}, \bar{\mu}, \bar{T})$ is a (generalized) Bernoulli shift if there exists a sub- $\sigma$ algebra $\Sigma_{0} \subset \bar{\Sigma}$ which is an independent generator for $\bar{T}$ : the sequence $\Sigma_{0}, \bar{T} \Sigma_{0}, \bar{T}^{2} \Sigma_{0}, \ldots$ forms an independent sequence of $\sigma$-algebras [17], and $\bigvee_{j=-\infty}^{\infty} \bar{T}^{j} \Sigma_{0}$ (the smallest $\sigma$-algebra containing all of the $\left.\bar{T}^{i} \Sigma_{0}\right)=\bar{\Sigma}(\bmod 0)$ (see [13]). For $X_{0} \in \Sigma$ we denote by $\Sigma_{X_{0}}$ the smallest $\sigma$-algebra for which the functions $f$ measurable in $X_{0}$ [i.e., $f$ is $(\bar{\Sigma})$ measurable and $f(\bar{x})$ $=f\left(\bar{x} \cap \bar{X}_{0}\right)$ for $\left.\bar{x} \in \bar{X}\right]$ are measurable. If $\left\{T^{n} X_{0}\right\}$ is a family of disjoint sets with $\bigcup_{n=-\infty}^{\infty} T^{n} X_{0}=X$, then $\Sigma_{X_{0}}$ is an independent generator for $\bar{T}$.

It is precisely in this way that the time evolution of the infinite ideal gas may be shown to form a $B$-flow ${ }^{7}$. We may identify the infinite ideal gas with the Poisson system $\left(\bar{X}^{I}, \bar{\Sigma}^{I}, \bar{\mu}^{I},\left\{\bar{T}_{t}^{I}\right\}\right)$ built over $\left(X^{I}, \Sigma^{I}, \mu^{I},\left\{T_{t}^{I}\right\}\right)$, where $X^{I}=\mathbb{R}^{v} \times \mathbb{R}^{v}, \Sigma^{I}=$ the class of Lebesgue sets of $\mathbb{R}^{v} \times \mathbb{R}^{v}, d \mu^{I}$ $=(\beta / 2 \pi)^{v / 2} e^{-1 / 2 \beta v^{2}} d \boldsymbol{q} d \boldsymbol{v}$, and $T_{t}^{I}(\boldsymbol{q}, \boldsymbol{v})=(\boldsymbol{q}+\boldsymbol{v} t, \boldsymbol{v})$ for $(\boldsymbol{q}, \boldsymbol{v}) \in \mathbb{R}^{v} \times \mathbb{R}^{v}$. $(v$ is the dimension of the physical space.) If we let $X_{0}^{I}=\left\{(\boldsymbol{q}, \boldsymbol{v}) \in X^{I} \mid q_{1}+v_{1} t=0\right.$, for some $0<t<1\}$, we find that $\Sigma_{X_{0}^{I}}$ is an independent generator for $\bar{T}\left(\equiv \bar{T}_{1}\right)^{8}$. We remark that, as one would expect, there exist Poisson systems for which no Bernoulli construction is possible, which possess, nontheless, very strong ergodic properties [1]; in fact the failure to permit a Bernoulli construction is an indication of "better thermodynamic behavior" than would otherwise be present.

We now describe a class of systems which may, be regarded as the infinite volume limits of periodic systems of independent particles whose one particle finite volume components form $K$-systems [1]. We will say that the Poisson system $(\bar{X}, \bar{\Sigma}, \bar{\mu}, \bar{T})$ is of periodic-K-type if the following are true:

1) There exists an isomorphism $\varphi$ from $\left(X_{0}, \Sigma_{0}, \mu_{0}\right) \times\left(\mathbb{Z}^{v}, \Sigma_{\mathbb{Z}} v, \mu_{\mathbb{Z}^{v}}\right)$ $\left[\left(X_{0}, \Sigma_{0}, \mu_{0}\right)\right.$ is a totally finite measure space and $\left(\mathbb{Z}^{v}, \Sigma_{\mathbb{Z}} v, \mu_{\mathbb{Z}^{v}}\right)$ is the $v$-dimensional lattice $\left\{\left(i_{1}, \ldots, i_{v}\right) \mid i_{k} \in \mathbb{Z}, 1 \leqq k \leqq v\right\}$ with counting measure $]$ to $(X, \Sigma, \mu)$ such that the translation group $\mathscr{S}\left(\equiv \varphi\left(\mathscr{S}_{\mathbb{Z}^{v}}\right)\right)$ induced by the translations $S_{l}: \mathbb{Z}^{v} \rightarrow \mathbb{Z}^{v}, S_{l} k=k+l\left(k, l \in \mathbb{Z}^{v}\right)$, commutes with $T$.

\footnotetext{
${ }^{7}$ Ornstein has shown that $\left\{T_{t}\right\}$ is a $B$-flow ( $B$-shift for all $t$ ) if $T_{1}$ is a Bernoulli shift [7].

8 The infinite ideal gas is, of course, also a $B$-system under translations, as are all the systems whose space-time ergodic properties we shall consider.
} 
2) There exists a sequence of rectangles $R_{i}$ whose volumes approach infinity, such that the induced automorphisms $T_{R_{i}}$ are $K$-automorphisms ${ }^{9}$. By a rectangle $R$ we mean a set of the form $\varphi\left(X_{0} \times \mathbb{Z}_{R}^{v}\right)$, where $\mathbb{Z}_{R}^{v}$ is a (finite) "rectangle" in $\mathbb{Z}^{v}$, by the volume of $R$ we mean \# $\left(\mathbb{Z}_{R}^{v}\right)$, and by $T_{R}$ we mean the automorphism of $R$ (equipped with the probability measure induced by $\mu$ ) induced by the natural map $\mathbb{Z}^{v} \rightarrow \mathbb{Z}_{R}^{v}$ (periodic boundary conditions on $R$ ).

3) $T\left(R_{0}\right)$ is bounded. Here $R_{0}=\varphi\left(X_{0} \times\{0\}\right)$ and by bounded we mean contained in a rectangle.

4) $T_{R_{0}}$ has finite entropy.

The base system $(X, \Sigma, \mu, T)$ will be called a base-periodic- $K$-system.

A Poisson system with base satisfying 1 ) will be said to be of periodic type or periodic (with periodic representation given by $\varphi$ ), while a Poisson system with base satisfying 1) and 3) will be said to be of periodicbounded-type.

The measure space of the infinite ideal gas (in $v$ dimensions) possesses the commutative group of automorphisms generated by the flows $\left\{\bar{T}_{t}^{I}\right\}$ and $\left\{\bar{S}_{j, s}\right\}, 1 \leqq j \leqq v$, the space translations. If we identify $\mathscr{S}$ with the group generated by the automorphisms $S_{j} \equiv S_{j, 1}, 1 \leqq j \leqq v$, and $T$ with $T_{1}$, we see that the ideal gas is periodic. However, corresponding to the possibility of the occurrence of arbitrarily large velocities, it is not of periodic-bounded-type, though it does satisfy a condition which will be just as useful for our purposes. Denoting the group generated by $\mathscr{S}$ and $T(\overline{\mathscr{S}}$ and $\bar{T})$ by $\mathscr{I}(\overline{\mathscr{I}})$, we will say that a periodic system $(\bar{X}, \bar{\Sigma}, \bar{\mu}, \bar{T})$ is of periodic- $\sigma$-bounded type if there exists an increasing sequence $\left\{X_{n}\right\}$ of $\mathscr{I}$-invariant $\Sigma$-subsets of $X$ such that $T\left(X_{n} \cap R_{0}\right)$ is bounded for any $n$ and $\bigcup_{n} X_{n}=X$. It is clear that the infinite ideal gas is of periodic$\sigma$-bounded type.

Given any measurable partition $[13,14] \zeta$ of $(X, \Sigma, \mu)$ we denote by $\bar{\zeta}$ the (measurable) partition of $(\bar{X}, \bar{\Sigma}, \bar{\mu})$ according to the number of particles in the elements of $\zeta$. In [1] it is shown that a system of periodic$K$-type is a $K$-system with a $K$-partition $\bar{\zeta}$ such that $\zeta$ is a base- $K$ partition (see [1]). In particular,

a) $\zeta$ is invariant under $\mathscr{S} .(\mathscr{S} \zeta=\zeta$.)

b) $\zeta$ is finer than $Q_{0}$, the partition of $X$ induced by the partition of $\mathbb{Z}^{v}$ into its points. $\left(\zeta \geqq Q_{0} ; Q_{0}=\left\{\varphi\left(X_{0} \times\{i\}\right)\right\}, i \in \mathbb{Z}^{v}\right.$.)

Let $(X, \Sigma, \mu, T),\left(X_{0}, \Sigma_{0}, \mu_{0}\right)$, and $\varphi$ be as in 1) and let $T_{0}$ be the automorphism of $X_{0}$ obtained from $T_{R_{0}}$ by the identification of $R_{0}$ $\left(\equiv \varphi\left(X_{0} \times\{0\}\right)\right)$ with $X_{0}$. Let $X_{0}$ possess a measurable partition $\left\{X_{\alpha}\right\}$ whose elements $X_{\alpha}$ are invariant under $T_{0}$, let $\left\{\mu_{\alpha}\right\}$ be the canonical system of measures [13] associated with $\left\{X_{\alpha}\right\}$ [and $\left(X_{0}, \Sigma_{0}, \mu_{0}\right)$ ], and let

\footnotetext{
${ }^{9}$ If $\left(X^{\prime}, \Sigma^{\prime}, \mu^{\prime}, T^{\prime}\right)$ is a $K$-system, we say that $T^{\prime}$ is a $K$-automorphism.
} 
$T_{\alpha}$ be the restriction of $\varphi^{-1} \circ T \circ \varphi$ to $X_{\alpha} \times \mathbb{Z}^{v}$. If $\left\{\left(X_{\alpha} \times \mathbb{Z}^{v}, T_{\alpha}\right)\right\}$ (with the obvious measure) forms a family of measurably related base-periodic- $K$ systems (with periodic representation given by the identity mapping), we will say that $(\bar{X}, \bar{\Sigma}, \bar{\mu}, \bar{T})$ is of periodic- $\sigma$-K-type. By measurably related we mean that there exists a family $\left\{\zeta_{\alpha}\right\}$ such that $\zeta_{\alpha}$ is a base- $K$-partition for $\left(X_{\alpha}, \mu_{\alpha}, T_{\alpha}\right)$ and $\zeta=\bigcup_{\alpha} \zeta_{\alpha}$ is measurable. It is shown in [1] that $\bar{\zeta}$ is a $K$-partition, so that a system of periodic- $\sigma$ - $K$-type has a $K$-partition with properties identical to a) and $b$ ) above for a partition of systems of periodic- $K$-type.

It is a consequence of the work of Sinai $[18,6]$ that the dynamical system representing an infinite gas of independent particles moving with the same speed in a two dimensional (nonpathological) periodic array of (fixed) convex barriers (from which the particles undergo elastic collisions) is of periodic- $K$-type [1]. However, the Lorentz gas, in which the particles have a Maxwellian velocity distribution-corresponding to the thermodynamic limit of a grand canonical ensemble - and which is the more natural object from the standpoint of statistical mechanics, is not of periodic- $K$-type. It is, however, of periodic- $\sigma$ - $K$-type [1].

\section{Properties of Generalized Dynamical Systems ${ }^{10}$}

We consider a (generalized) abstract dynamical system $(X, \Sigma, \mu, \mathscr{I})$, where $\mathscr{I}$ is generated by $n$ commuting automorphisms of the probability space $(X, \Sigma, \mu)$. To simplify the notation we explicitly treat only the case $n=2$. Let $(S, T)$ be a pair of automorphisms which generates $\mathscr{I}$. Every such pair determines a homomorphism from the group $\mathbb{Z}^{2}$ to $\mathscr{I}$, permitting the representation of the elements of $\mathscr{I}$ by the points of $\mathbb{Z}^{2}$. Though some properties will be formulated in terms of the pair $(S, T)$, they will, in fact, depend only upon $\mathscr{I}$, unless we explicitly indicate otherwise. However, we intend for the definitions which we shall give to be applicable to dynamical systems of the form $(X, \Sigma, \mu,\{S, T\})$, where $S$ and $T$ are commuting automorphisms possibly satisfying some relation such as $S=T$.

We will say that a sequence $\varrho_{n}$ of parallelograms in $\mathbb{Z}^{2}$ approaches infinity if the smallest of its dimensions (orthogonal distance between parallel sides) approaches infinity. We will denote by $N(\varrho)$ the number of points in the parallelogram $\varrho$. For any measurable partition $P$ and $g \in \mathscr{I}$, we let

$$
P_{g}=\bigvee_{j=-\infty}^{\infty} g^{j} P, \quad P_{g}^{-}=\bigvee_{j=-\infty}^{-1} g^{j} P, \quad \text { and } \quad P_{\mathscr{I}}=\bigvee_{g^{\prime} \in \mathscr{I}} g^{\prime} P^{11} .
$$

${ }^{10}$ For a general reference for much of the material of this section, see Conze [11].

11 We use the notation $(\vee, \wedge, \leqq \geqq \varepsilon, v, \uparrow \downarrow)$ of $[14,13]$. 
We denote by $\mathscr{W}$ the orthogonal complement in $L^{2}(\mu)$ of the constants. (The generalization of the properties which we shall describe to the case in which the group $\mathscr{I}$ is generated by two flows $\left\{S_{s}\right\}$ and $\left\{T_{t}\right\}$ parallel the corresponding generalization from properties of a discrete dynamical system to properties of a flow.)

a) Ergodicity. $(X, \Sigma, \mu, \mathscr{I})$ is ergodic if all $A \in \Sigma$ which are invariant under $\mathscr{I}^{12}$ are such that $\mu(A)=0$ or $\mu(A)=1$. As in the case of a one parameter group, we have that if $(X, \Sigma, \mu, \mathscr{I})$ is ergodic, and only then,

$$
\begin{array}{r}
\lim _{n \rightarrow \infty} 1 / N\left(\varrho_{n}\right) \sum_{g \in \varrho_{n}} f(g x)=\lim _{n \rightarrow \infty} 1 / N\left(\varrho_{n}\right) \sum_{(k, l) \in \varrho_{n}} f\left(S^{k} T^{l} x\right) \\
=\int d \mu f, \quad \text { a.e., }
\end{array}
$$

for $\varrho_{n}$ a sequence of parallelograms approaching infinity, $f \in L^{1}(\mu)$, and $x \in X$. Ergodicity with respect to $\mathscr{I}$ is clearly a weaker property than, say, ergodicity with respect to $T$. It is the only such property which we shall encounter.

b) Mixing. $(X, \Sigma, \mu, \mathscr{I})$ is mixing if

$$
\lim _{g \rightarrow \infty} \mu(g A \cap B)=\mu(A) \mu(B)
$$

for all $A, B \in \Sigma^{13}$. Since convergence to infinity in $\mathbb{Z}^{2}$ is invariant under automorphisms of $\mathbb{Z}^{2}$, the definition does not depend on the choice of generators.

c) Countable Lebesgue Spectrum. $(X, \Sigma, \mu, \mathscr{I})$ has countable Lebesgue spectrum if there exists a family $\left\{f_{(j, k)}^{i}\right\}, i \in \mathbb{Z},(j, k) \in \mathbb{Z}^{2}$, of functions forming an orthonormal basis of $\mathscr{W}$ and satisfying

$$
U_{S^{n} T^{m}} f_{(j, k)}^{i}=U_{S}^{n} U_{T}^{m} f_{(j, k)}^{i}=f_{(j+n, k+m)}^{i}
$$

for all $(j, k) \in \mathbb{Z}^{2},(n, m) \in \mathbb{Z}^{2}$, and $i \in \mathbb{Z}^{14}$. Just as in the case of a one parameter group, a system with Lebesgue spectrum is mixing.

d) Entropy. The entropy of a group $\mathscr{I}$ is defined in a manner completely analagous to the definition of the entropy of an automorphism $T^{15}$. We need mention only that the entropy of a (countable) measurable partition $P$ relative to the group $\mathscr{I}$ is defined by

$$
h(P, \mathscr{I})=\lim _{n \rightarrow \infty} 1 / N\left(\varrho_{n}\right) H\left(\bigvee_{g \in \varrho_{n}} g P\right),
$$

${ }^{12} A$ is invariant under $\mathscr{I}$ if and only if $g A=A$ for all $g \in \mathscr{I}$.

13 By $g \rightarrow \infty$ we mean in the sense of the natural locally compact topology on $\mathbb{Z}^{2}$. The generalization to an arbitrary locally compact topological group is immediate.

${ }^{14}$ If $F$ is an automorphism of $(X, \Sigma, \mu)$ we denote by $U_{F}$ the unitary on $L^{2}(\mu)$ induced by $F: U_{F} f=f \circ F$.

15 For the definition and properties of entropy, see $[8,10,14]$. 
where $\left\{\varrho_{n}\right\}$ is a sequence of parallelograms approaching infinity. The limit is independent of the particular sequence of parallelograms, and, consequently, $h(P, \mathscr{I})$ is independent of the choice of generators. In much the same way as for a single automorphism, one verifies that if $H(P)<\infty$

$$
h(P, \mathscr{I})=H\left(P \| P_{S}^{-} \vee\left(P_{S}\right)_{T}^{-}\right)^{16} .
$$

We will call $P_{S}^{-} \vee\left(P_{S}\right)_{T}^{-}$the past of $P$ relative to $(S, T)$ and denote it by $P_{\mathscr{I}}^{-17}$. We also note that if $Q$ is a generator for $\mathscr{I}$ with finite entropy [i.e., $Q_{\mathscr{I}}=\varepsilon^{18}(\bmod 0)$, and $\left.H(Q)<\infty\right]$, we have for the entropy of $\mathscr{I}$

$$
h(\mathscr{I})=\sup _{P \text { finite }} h(P, \mathscr{I})=\sup _{H(P)<\infty} h(P, \mathscr{I})=h(Q, \mathscr{I}) .
$$

Finally, we say that $(X, \Sigma, \mu, \mathscr{I})$ has completely positive entropy if $h(P, \mathscr{I})>0$ for all nontrivial partitions $P$. If $(X, \Sigma, \mu, \mathscr{I})$ has completely positive entropy it is mixing.

e) K-Systems. We define the $K$-system property for an ordered pair of (commuting) automorphisms $(S, T)$ rather than for the group $\mathscr{I}$ which they generate. Insofar as space translations and time evolution play rather different roles in statistical mechanics, this distinction is quite appropriate. The key to the generalization is the extension of the natural ordering on $\mathbb{Z}$, on the structure of which the notion of $K$ system for an automorphism is implicitly based, to an ordering on $\mathbb{Z}^{2}$. We write $(n, m) \leqq(p, q)$ if $m<q$, or if $m=q$ and $n \leqq p$. We will say that $(X, \Sigma, \mu,(S, T))$ is a $K$-system if there exists a measurable partition $\zeta$ such that

1) $\zeta$ is increasing:

$$
S^{n} T^{m} \zeta \leqq S^{p} T^{q} \zeta(\bmod 0) \quad \text { if } \quad(n, m) \leqq(p, q),
$$

2) $\bigvee_{(n, m) \in \mathbb{Z}^{2}} S^{n} T^{m} \zeta=\varepsilon(\bmod 0)$,

3) $\bigwedge_{m} S^{-m} \zeta=T^{-1} \zeta_{S}(\bmod 0)$,

4) $\bigwedge_{n} T^{-n} \zeta_{S}=v^{19}(\bmod 0)$.

Note that if $(X, \Sigma, \mu,(S, T))$ is a $K$-system, $(X, \Sigma, \mu, T)$ possesses an $S$-invariant $K$-partition, namely $\zeta_{S}$. If $(S, T)$ forms a $K$-system, the group $\mathscr{I}$ generated by $S$ and $T$ has completely positive entropy and, by essentially

${ }^{16}$ We use the notation $H(\alpha \| \beta)$ for the conditional entropy of the measurable partition $\alpha$ relative to the measurable partition $\beta$.

${ }^{17}$ There are, of course, seven other possible choices of a "past" of $P$ which we could insert in the above relation in place of $P_{\mathscr{g}}^{-}$without altering its validity. For the systems which we consider we make the "natural" choice for the pair of generators $(S, T)$.

${ }^{18} \varepsilon$ is the partition into individual points.

${ }^{19} v$ is the trivial partition of $X$; its sole element is $X$ itself. 
the same argument as for a single automorphism, countable Lebesgue spectrum.

f) Bernoulli Systems. $(X, \Sigma, \mu, \mathscr{I})$ is a Bernoulli system if there exists a measurable partition $P$ which is a generator for $\mathscr{I}$ such that $\{g P\}, g \in \mathscr{I}$, is an independent family of partitions ${ }^{20}$. If $(X, \Sigma, \mu, \mathscr{I})$ is Bernoulli, then $(X, \Sigma, \mu,(S, T))$ is a $K$-system for any pair of generators $(S, T):$ if $P$ is an independent generator for $\mathscr{I}$,

$$
\zeta=\bigvee_{(n, m) \leqq(0,0)} S^{n} T^{m} P
$$

is a $K$-partition for $(S, T)$. Ornstein's theorems can be extended to this generalized framework [12].

g) Formula of Abramov. If $\mathscr{I}$ is generated by $\left\{S_{t}\right),\left\{T_{t}\right\}, \ldots\left\{R_{t}\right\}$, $n$ commuting groups depending continuously on a real parameter, a generalization of the formula of Abramov $\left(h\left(T_{t}\right)=|t| h\left(T_{1}\right)\right)$ can be obtained [11]. Let $\Gamma$ be the subgroup of $\mathscr{I}$ generated by $S_{1}, T_{1}, \ldots, R_{1}$. If we regard $\mathscr{I}$ as a real vector space with basis $S_{1}, T_{1}, \ldots, R_{1}$, we can operate on $\Gamma$ by a real $n \times n$ matrix $M$ to obtain a subgroup $\Gamma_{M}$. Then

$$
h\left(\Gamma_{M}\right)=|\operatorname{det}(M)| h(\Gamma) .
$$

\section{Invariance of Space-Time Ergodic Properties under Galilean Transformations}

For many applications to classical statistical mechanics it suffices to consider a dynamical system $(X, \Sigma, \mu, \mathscr{I})$, where $\mu$ is an equilibrium state for a $v$-dimensional infinite system of particles and $\mathscr{I}$ is the group generated by $S_{1,1}, \ldots, S_{v, 1}$, the unit space translations, and $T_{1}$, the unit time evolution. In this framework we will consider only Galilean transformations determined by integral velocities. Most of the results can be extended to arbitrary Galilean and Lorentz transformations in the case where $\mathscr{I}$ is generated by the complete (continuous) space-time group.

Accordingly, let $(X, \Sigma, \mu)$ be a translation invariant equilibrium state of a one dimensional (for notational convenience) system of infinitely many particles. Let $T_{t}$ denote its time evolution and $S_{s}$ the spatial translations. We can describe a trajectory induced by $T_{t}$ by specifying a family $\left\{q_{i}(t)\right\}, t \in \mathbb{R}$, of functions representing the time evolution of the positions of the individual particles, labelled arbitrarily.

\footnotetext{
${ }^{20}$ By virtue of the natural correspondence between measurable partitions (mod 0 ) and sub- $\sigma$-algebras $(\bmod 0)($ see $[14,13])$, this definition is a generalization of the definition given in Section 2 for a single automorphism. For any measurable partition $\alpha$, we denote by $\hat{\alpha}$ the corresponding sub- $\sigma$-algebra, and conversely.
} 
A Galilean transformation $G_{v}$ at velocity $v$ transforms a trajectory $\left\{q_{i}(t)\right\}$ into a trajectory $\left\{q_{i}(t)-v t\right\}=G_{v}\left(\left\{q_{i}(t)\right\}\right) . G_{v}$, of course, also transforms the velocities according to $G_{v}\left(\left\{v_{i}(t)\right\}\right)=\left\{v_{i}(t)-v\right\}$. Thus, in an obvious manner, $G_{v}$ transforms the system $\left(X, \Sigma, \mu,\left(S_{s}, T_{t}\right)\right)$ into the system $\left(X^{\prime}, \Sigma^{\prime}, \mu^{\prime},\left(S_{s}^{\prime}, T_{t}^{\prime}\right)\right)$. From the standpoint of our abstract framework we can identify the latter with $\left(X, \Sigma, \mu,\left(S_{s}, T_{t} S_{v t}\right)\right)$, so that the effect of $G_{v}$ may be regarded as the replacement of the pair $\left(S_{s}, T_{t}\right)$ by the pair $\left(S_{s}, T_{t} S_{v t}\right)$, or, in the discrete case, assuming $v$ to be an integer, $\left(S_{1}, T_{1}\right)$ by $\left(S_{1}, T_{1} S_{1}^{v}\right)^{21,22}$. Consequently, those properties which depend upon only the group $\mathscr{I}$ are invariant under Galilean transformations: $S$ and $T S^{v}$ generate the same group as do $S$ and $T$. Furthermore, the concept of mixing for the pair $(S, T)$, which depends upon the notion of convergence to infinity in $\mathbb{Z}^{2}$, is invariant under $G_{v}$, since the automorphism of $\mathbb{Z}^{2}$ induced by the replacement $(S, T) \rightarrow\left(S, T S^{v}\right)$ leaves such convergence invariant. Finally, the concept of $K$-system, which depends upon the ordered pair $(S, T)$ and, specifically, upon the ordering of $\mathbb{Z}^{2}$ which the pair induces, is invariant under $G_{v}$, since $\left(S, T S^{v}\right)$ induces the same ordering as does $(S, T)$.

\section{Space-Time Ergodic Properties of the Ideal Gas}

We proceed to the investigation of the space-time ergodic properties of periodic systems and, in particular, of the equilibrium states of systems of infinitely many independent particles. We will deal with one-dimensional systems; the results and arguments can easily be adapted to several spatial dimensions. In this section we show that though the ideal gas has countable Lebesgue spectrum even in the space-time framework, it is not a $K$-system for the pair $(\bar{S}, \bar{T})$, since its space-time entropy vanishes.

We first exhibit an example of two systems identical from the standpoint of the time evolution considered by itself which are distinguishable within the space-time framework. A system identical to the infinite ideal gas except that, instead of a Maxwellian velocity distribution, all particles move with unit velocity (to the right) is clearly a Bernoulli flow under the time evolution and hence isomorphic to the ideal gas. However, since the time evolution and the space translation act in the same manner on the phase space of this system, it is not "jointly" mixing; in fact, $\bar{S}^{n} \bar{T}^{n}$ (for the appropriate choice of $\bar{S} \equiv \bar{S}_{1}$ ) is the identity transformation for all $n$, though $(n, n) \rightarrow \infty$ in $\mathbb{Z}^{2}$.

21 The effect of a Lorentz transformation would be the replacement, as well, of $S_{s}$ by some $S_{\alpha s} T_{\beta s}, \alpha, \beta \in \mathbb{R}$, since under a Lorentz transformation both the space and time axes become obliquely oriented with respect to the original axes.

22 Henceforth, we will write $(S, T)$ for $\left(S_{1}, T_{1}\right)$, etc. 
We denote by $S_{s}^{I}$ the spatial translation on the one particle ideal gas phase space $X^{I}\left[\right.$ i.e., $\left.S_{s}^{I}(q, v)=(q-s, v),(q, v) \in \mathbb{R} \times \mathbb{R}\right]$ and by $\bar{S}_{s}^{I}$ the flow on $\left(\bar{X}^{I}, \bar{\mu}^{I}\right)$ induced by $S_{s}^{I}$. That $\left(\bar{X}^{I}, \bar{\mu}^{I},\left\{\bar{S}_{s}^{I}, \bar{T}_{t}^{I}\right\}\right)$ is not isomorphic to the system described above is a consequence of the simple

Proposition 5.1. $\left(\bar{X}^{I}, \bar{\Sigma}^{I}, \bar{\mu}^{I},\left\{\bar{S}_{s}^{I}, \bar{T}_{t}^{I}\right\}\right)$ is mixing.

Proof. The proposition follows (see [1]) from the observation that if $A, B \in \Sigma^{I}$ are bounded subsets of $\mathbb{R}^{2}$,

$$
\lim _{(s, t) \rightarrow \infty} \mu^{I}\left(\left(S_{s}^{I} T_{t}^{I} A\right) \cap B\right)=0 .
$$

The space-time ergodic properties of the ideal gas are, in fact, somewhat stronger than mixing:

Proposition 5.2. $\left(\bar{X}^{I}, \bar{\Sigma}^{I}, \bar{\mu}^{I}, \bar{I}^{I}\right)^{23}$ has countable Lebesgue spectrum.

Proof. By virtue of the fact, the proof of which will be given in Appendix I, that if $(\bar{X}, \bar{\Sigma}, \vec{\mu})$ is a Poisson probability space, there exists an isomorphism from $L^{2}(\bar{\mu})$ onto $\mathscr{J}$, the boson Fock space of $L^{2}(\mu)$, which carries any induced unitary $U_{\bar{T}}$ onto the unitary on $\mathscr{J}$ whose restriction to the $n$-particle subspace is $U_{T}^{\otimes n}\left(\equiv U_{T} \otimes \cdots \otimes U_{T}\right)$, it suffices to show that $\left(X^{I}, \Sigma^{I}, \mu^{I}, \mathscr{I}^{I}\right)$ has Lebesgue spectrum.

Let $U_{s}=U_{S_{S}^{I}}$ and $V_{t}=U_{T_{t}^{I}}$. Then for $f \in L^{2}\left(\mu^{I}\right)\left(\equiv L^{2}\left(\mathbb{R}^{2}, e^{-v^{2}} d q d v\right)\right)$ we have

$$
U_{s} V_{t} f(q, v)=f(q-s+v t, v) .
$$

Let $\psi \circ \varphi$ be the isomorphism of $L^{2}\left(\mathbb{R}^{2}, e^{-v^{2}} d q d v\right)$ onto $L^{2}\left(\mathbb{R}^{2}, e^{-w^{2} / k^{2}} /|k|\right.$ $\cdot d k d w)$ obtained by composing a $q$-Fourier transform with the isomorphism induced by the change of variables $(k, v) \rightarrow(k, w)=(k, k v)$. This isomorphism carries $U_{s}$ and $V_{t}$ onto $U_{s}^{\prime}$ and $V_{t}^{\prime}$, respectively, where

$$
U_{s}^{\prime} V_{t}^{\prime} \bar{f}(k, w)=e^{-i(k s-w t)} \bar{f}(k, w)
$$

for $\bar{f} \in L^{2}\left(\mathbb{R}^{2}, e^{-w^{2} / k^{2}} /|k| d k d w\right)$. We thus have a representation of $U_{s} V_{t}$ as the operator of multiplication by $e^{-i(k s-w t)}$ on $L^{2}\left(\mathbb{R}^{2}, d \tilde{\mu}(k, w)\right)$ with $\tilde{\mu}$ a measure on $\mathbb{R}^{2}$ equivalent to Lebesgue measure, from which the proposition easily follows.

Fortunately, countable Lebesgue spectrum is the strongest space-time ergodic property which we shall find that the ideal gas possesses. In [1], the nonlocal nature of the dissipation of disturbances of the ideal gas was alluded to, as a sympton of which we might regard the manifestly non translation invariant nature of its $K$-partition. Since a space-time $((\bar{S}, \bar{T})) K$-system must possess, in particular, a translation invariant $K$-partition for the time evolution $\bar{T}$, and since it appears implausible that the time evolution of the ideal gas should possess such a $K$-partition,

${ }^{23} \bar{I}^{I}$ is, of course, the group of space-time translations of $\bar{X}^{I}$, induced by the group $\mathscr{I}^{I}$ of space-time translations of $X^{I}$. 
we expect it to fail to be a $K$-system for $\left(\bar{S}^{I}, \bar{T}^{I}\right)$. Rather than verifying that no such partition exists, we will show that the space-time entropy of the ideal gas vanishes. Since $K$-systems have completely positive entropy, this will imply the desired result.

Theorem 5.3. $h\left(\overline{\mathscr{I}}^{I}\right)=0$, so that $\left(\bar{X}^{I}, \bar{\Sigma}^{I}, \bar{\mu}^{I},\left(\bar{S}^{I}, \bar{T}^{I}\right)\right)$ is not a $K$-system.

Proof. We compute $h\left(\overline{\mathscr{I}}^{I}\right)$ by finding a partition $P^{I}$ of finite entropy such that

$$
P_{\bar{I}}^{I^{-}}=\varepsilon(\bmod 0) .
$$

Then, since $P^{I}$ will be a generator for $\bar{I}^{I}$, we will have

$$
h\left(\overline{\mathscr{I}}^{I}\right)=h\left(P^{I}, \overline{\mathscr{I}}^{I}\right)=H\left(P^{I} \| P_{\bar{I}^{I}}^{I-}\right)=0^{24} .
$$

For any $\bar{x} \in \bar{X}^{I}$ and $m \in \mathbb{Z}$, let $\left(q_{1}, v_{1}\right), \ldots,\left(q_{n}, v_{n}\right)$ be the coordinates of the particles of $\bar{x}$ in $[m, m+1) \times \mathbb{R}$. If $q_{i} \leqq q_{j}$ for $i<j$, we will say that the particles in $[m, m+1)$ are labeled in natural order, and that the particle with coordinates $\left(q_{i}, v_{i}\right)$ has index $i$ in $[m, m+1)$. If a particle of $\bar{T}^{I} \bar{x}$ with coordinates $(q+v, v)$ has index $j$ in $[m, m+1)$, we will often say that the particle of $\bar{x}$ with coordinates $(q, v)$ has index $j$ in $[m, m+1)$ at $t=1$.

We choose for $P^{I}$ the partition whose atoms are of the form $P_{\left\{n ;\left(m_{1}, k_{1}\right), \ldots,\left(m_{i}, k_{i}\right), \ldots,\left(m_{n}, k_{n}\right)\right\}}=\left\{\bar{x} \in \bar{X}^{I} \mid N_{[0,1] \times \mathbb{R}}(\bar{x})=n\right.$, and the particle of $\bar{x}$ with index $i$ in $[0,1)$ has index $k_{i}$ in $\left[m_{i}, m_{i}+1\right)$ at $t=1$, for all $\left.i=1, \ldots n\right\}$. The theorem now follows from two lemmas:

Lemma 5.4. $P_{\bar{Y}}^{I-}=\varepsilon(\bmod 0)$.

Lemma 5.5. $H\left(P^{I}\right)<\infty$.

Proof of Lemma 5.4. It suffices to show that

$$
\tilde{P}^{I}=\left(P_{\bar{S}^{I}}^{I}\right)_{\overline{\bar{T}}}^{I}=\varepsilon(\bmod 0),
$$

which we will do by showing that $\tilde{P}^{I}$ contains sufficient information to determine $(\bmod 0)$ all of the coordinates $\left\{\left(q_{i}, v_{i}\right)\right\}$ of the particles of a point $\bar{x} \in \bar{X}^{I}$, and hence $\bar{x}$ itself. Now $P_{\bar{S}^{I}}^{I}$ determines the number of particles in each unit cell $[i, i+1) \times \mathbb{R}$ of $\mathbb{R}^{2}$, as well as the immediate future of each particle to the extent of requiring, for example, that the particle which at $t=0$ has index $j$ in $[k, k+1)$ have index $l$ in $[m, m+1)$ at $t=1^{25}$. Similarly $\left(\bar{T}^{I}\right)^{-1} P \bar{S}^{I}$ provides analogous information for times $t=1$ and $t=2$, and the index information determined by $P^{I}$ enables us to unambiguously trace every particle from $t=0$ to $t=2$ with respect to the partition of $X^{I}$ into unit cells, using the information determined by

${ }^{24}$ See [11].

${ }^{25}$ By the information (about $\bar{x}$ ) determined by a partition $P$ we mean the information we would have if we knew of which element of $P \bar{x}$ is a member. 
$P_{\bar{S}^{I}}^{I} \vee\left(\bar{T}^{I}\right)^{-1} P_{\bar{S}^{I}}$. Proceeding in this way, we see that $\tilde{P}$ determines the trajectory of each particle of $\bar{x} \in \bar{X}^{I}$, with respect to unit cells, from $t=0$ to $t=\infty$, so that the velocities of all the particles are uniquely determined. The Jacobi theorem for the irrational rotation of the circle implies that the positions of particles with irrational velocity are also determined by $\tilde{P}^{I}$, since for irrational $v$ the sequence $q, q+v, q+2 v, \ldots$ is dense in $\mathbb{R}(\bmod 1)$. Finally, since the Maxwellian distribution $\mu_{\beta}=\sqrt{\beta / 2 \pi} e^{-1 / 2 \beta v^{2}}$ assigns zero measure to the set of rational velocities, we have that

$$
\tilde{P}^{I}=\varepsilon(\bmod 0) .
$$

The proof of Lemma 5.5, which depends upon elementary estimates, will be given in Appendix II.

The method of proof of Theorem 5.3 is an extension to an infinite system of particles of the method of proof for the vanishing of the entropy of a finite ideal gas [14]. We also remark that a similar argument, using, in particular, a partition analogous to $P^{I}$, can be used to show that an infinite one-dimensional system of hard rods has vanishing space-time entropy (Aizenman, private communication). Finally, we observe that though the above argument works only for a velocity distribution assigning zero measure to rational velocities, the theorem is valid for an arbitrary velocity distribution, since we can always change the time scale in such a way that the argument is applicable and then apply the formula of Abramov (Section 3g) to obtain that

$$
h(\overline{\mathscr{I}})=\tau_{0} h\left(\overline{\mathscr{I}}_{\tau_{0}}\right)=0,
$$

where $\overline{\mathscr{I}}_{\tau_{0}}$ is the group generated by unit space and time translations corresponding to a change of time scale by the appropriate factor $\tau_{0}$.

\section{Space-Time Ergodic Properties of Periodic Systems}

We will deal explicitly with periodic systems with one spatial dimension $(v=1)$; the results and proofs extend readily to arbitrary $v$, at the expense of a somewhat more cumbersome notation ${ }^{26}$. We denote by $S \in \mathscr{S}$ the unit translation of the base measure space $(X, \Sigma, \mu)$.

\section{a) Space-Time K-Systems}

Having shown that the ideal gas is not a $K$-system for $\left(\bar{S}^{I}, \bar{T}^{I}\right)$, we formally distinguish it from systems like the Lorentz gas by establishing

${ }^{26}$ We mention only that one must extend the lexicographical ordering, which will be used in several places, to a lattice of a larger number of dimensions. 
that such systems are, indeed, space-time $K$-systems. That such systems have $K$-partitions which are translation invariant strongly suggests that this is the case.

Theorem 6.1. If $(\bar{X}, \bar{\Sigma}, \bar{\mu}, \bar{T})$ is of periodic-K-type, then $(\bar{X}, \bar{\Sigma}, \bar{\mu},(\bar{S}, \bar{T}))$ is a K-system.

Proof. If we can express $\bar{\zeta}[\zeta$ a base $K$-partition of $(X, \Sigma, \mu, T)]$ in the form $\xi_{\bar{S}}$, where $\xi$ (a measurable partition) satisfies 1) and 3) of the definition of an $(S, T) K$-system (see Section $2 \mathrm{e}$ ), we will be done, since 2) and 4) follow from the $(\bar{T}) K$-properties of $\bar{\zeta}[1]$. We obtain such a $\xi$ by setting $\xi=\bar{T}^{-1} \bar{\zeta} \vee \bar{\zeta}^{+}$, where $\bar{\zeta}^{+}$is the restriction of $\bar{\zeta}$ to $\varphi\left(X_{0} \times \mathbb{Z}^{+}\right)$, i.e.,

$$
\bar{\zeta}^{+}=\bar{\zeta} \wedge \varepsilon^{+}
$$

where $\varepsilon^{+}$is the partition of $\bar{X}$ generated by functions measurable in $\varphi\left(X_{0} \times \mathbb{Z}^{+}\right)$. It is clear that $\bar{\zeta}=\xi_{\bar{s}}$ and that $\xi$ is increasing. The theorem thus follows from

Lemma 6.2. $\bigwedge_{n} \bar{S}^{-n} \xi=\bar{T}^{-1} \bar{\zeta}$.

Proof. The lemma follows, using Doob's martingale theorem (see [8] $)^{27}$, from the fact that for all $A \in \bar{\Sigma}$,

$$
\bar{\mu}\left(A \| \bigwedge_{n} \bar{S}^{-n} \xi\right)=\lim _{n \rightarrow \infty} \bar{\mu}\left(A \| \bar{S}^{-n} \xi\right)=\bar{\mu}\left(A \| \bar{T}^{-1} \bar{\zeta}\right), \quad \text { a.e. . }
$$

It sufficies to establish that the above equality is valid for $A$ a member of $\Sigma_{K}, K$ a bounded region of $X$. But for $A$ of this form we can find an $N$ such that for $n \geqq N$,

$$
\bar{\mu}\left(A \| \bar{S}^{-n} \xi\right)=\bar{\mu}\left(A \| \bar{T}^{-1} \bar{\zeta}\right), \quad \text { a.e. }
$$

we merely choose $N$ so large that

$$
\alpha \cap K \cap \varphi\left(X_{0} \times\{N, N+1, N+2, \ldots\}\right)=\phi,
$$

for all $\alpha \in T^{-1} \zeta$.

The preceding argument can be applied, essentially without modification, to systems of periodic- $\sigma$-K-type, such as the Lorentz gas ${ }^{28}$. We thus have

Theorem 6.3. System of periodic- $\sigma$-K-type (and in particular the Lorentz gas) form space-time K-systems.

27. For any probability measure $\mu$, any measurable set $E$, and any measurable partition $\alpha$, we denote by $\mu(E \| \alpha)=\mu(E \| \hat{\alpha})$ the conditional probability of $E$ with respect to $\alpha$ or $\hat{\alpha}$ (see [8] and Footnote 20).

28 The region $K$ must now be bounded in the "velocity" direction as well. 


\section{b) Space-Time Entropy}

We now investigate the space-time entropy of infinite systems of noninteracting particles. The proof of the vanishing of the spacetime entropy of the infinite ideal gas suggests - and can be generalized to showthat the space-time entropy of any infinite system whose finite volume one-particle components have vanishing time entropy vanishes as well. We will prove a stronger result.

A natural quantity to consider for infinite systems is the time entropy per unit volume. It would be nice if the space-time entropy of these systems could be so regarded. We will show that this is quite generally the case.

We first define the notion of the time entropy per unit volume of a periodic system $(\bar{X}, \bar{\Sigma}, \bar{\mu}, \bar{T})$. Recall that we have denoted by $T_{R}$ the restriction of $T$ to the rectangle $R$. Let $\left(\bar{R}, \overline{\Sigma \cap R}, \bar{\mu}_{R}, \bar{T}_{R}\right)$ be the Poisson system built over $\left(R, \Sigma \cap R, \mu_{R}, T_{R}\right)$, where $\mu_{R}$ is the restriction of $\mu$ to $\Sigma \cap R$, the sub- $\sigma$-algebra consisting of sets of the form $A \cap R, A \in \Sigma$. We define the $T$-entropy per unit volume by

$$
h_{1}(\bar{T})=\lim _{R \rightarrow \infty}(1 /\|R\|) h\left(\bar{T}_{R}\right),
$$

where $\|R\|$ is the volume of $R$ (see section 2$)^{29}$. We will first prove

Theorem 6.4. If $(\bar{X}, \bar{\Sigma}, \bar{\mu}, \bar{T})$ is of periodic-bounded-type, then

$$
H(\overline{\mathscr{J}})=h_{1}(\bar{T})=\mu\left(R_{0}\right) h\left(T_{R}\right)^{30}=(1 /\|R\|) h\left(\bar{T}_{R}\right) .
$$

Proof. For $P$ a partition of $R_{0}$ of finite entropy, let us denote by $Q_{0} P$ the "product" of $Q_{0}$ and $P$, i.e., the partition of $X$ whose elements are the translates of elements of $P$. Let $\tilde{h}(P, T)$ be given by

$$
\begin{aligned}
\tilde{h}(P, T) & =\lim _{n \rightarrow \infty}(1 / n) H\left(\left(\bigvee_{j=0}^{n-1} T^{j} Q_{0} P\right) \cap R_{0}\right) \\
( & \left.=H\left(\left(T Q_{0} P\right) \cap R_{0} \| \bigvee_{j=0}^{\infty} T^{-j} Q_{0} P\right) \cap R_{0}\right),
\end{aligned}
$$

where we are using the following notation: For any partition $P^{\prime}$ of $X$ we denote by $P^{\prime} \cap R$ the partition of $R$ induced by $P^{\prime}$ [with normalized measure $\mu_{R} / \mu(R)$ on $\left.R\right]$. Let $\tilde{h}(T)$ be given by

$$
\tilde{h}(T)=\sup _{P \text { finite }} \tilde{h}(P, T) .
$$

${ }^{29} R\left(R_{i}\right)$ will always be a rectangle. By $R_{i} \rightarrow \infty$, we mean that the length of the smallest side of $R_{i}$ approaches infinity. The above limit exists because $R \mapsto h\left(\bar{T}_{R}\right)$ is superadditive.

${ }^{30}$ When we write $h\left(T_{R}\right)$ we regard $T_{R}$ as an automorphism of the probability space $\left(R, \Sigma \cap R, \mu_{R} / \mu(R)\right)$. 
We will use the fact that in the same way as for a single automorphism [14], if $\left\{P_{n}\right\}$ is an increasing sequence of partitions of finite entropy with $\bigvee_{n} P_{n}=\varepsilon(\bmod 0)$, or even with $\bigvee_{n}\left(P_{n}\right)_{\mathscr{I}}=\varepsilon(\bmod 0)$, then

$$
h(\mathscr{I})=\lim _{n \rightarrow \infty} h\left(P_{n}, \mathscr{I}\right)
$$

for any (normalized) dynamical system $(X, \Sigma, \mu, \mathscr{I})$. A completely analgous result holds for $\tilde{h}$.

Theorem 6.4 easily follows from 3 lemmas:

Lemma 6.5. $\tilde{h}(T)=\lim _{R \rightarrow \infty} h\left(T_{R}\right)=h\left(T_{R}\right)$.

Lemma 6.6. $h(\overline{\mathscr{I}})=\mu\left(R_{0}\right) \tilde{h}(T)=\varrho \tilde{h}(T)$.

Lemma 6.7. $h\left(\bar{T}_{R}\right)=\mu(R) h\left(T_{R}\right)=\varrho\|R\| h\left(T_{R}\right)$.

Proof of Lemma 6.5. The first equality follows from the observation that for $R$ such that $R_{0} \subset R$ and $T\left(R_{0}\right) \subset R$ we have

indeed,

$$
\begin{gathered}
h\left(T_{R}\right)=\tilde{h}(T) ; \\
h\left(Q_{0} P \cap R, T_{R}\right)=\tilde{h}(P, T)
\end{gathered}
$$

for any finite entropy partition $P$ of $R_{0}$. The second equality holds because for any finite (measurable) ${ }^{31}$ partition $P$ of $R_{0}$. we have

$$
h\left(\left(Q_{0} P \vee T Q_{0} P\right) \cap R, T_{R}\right)=\tilde{h}\left(\left(Q_{0} P \vee T Q_{0} P\right) \cap R_{0}, T\right)^{32} .
$$

Proof of Lemma 6.6. We compute $h(\overline{\mathscr{I}})$ in a manner similar to the method used for computation of $h\left(\overline{\mathscr{I}}^{I}\right)$. For $P$ a finite partition of $R_{0}$, let $\overline{\bar{P}}$ be the partition of $\bar{X}$ constructed from $Q_{0} P$ in a manner analogous to the way in which $P^{I}$ was constructed from the partition of $X^{I}$ into unit cells: Let $P=\left\{P_{i}\right\}, i=1, \ldots, k$, and label the atoms of $Q_{0} P$ using the ordered pairs $(n, i), n \in \mathbb{Z}, i=1, \ldots, k ;(n, i)$ is the label of the "copy" of $P_{i}$ in $R_{n}=S^{n} R_{0}$. We order the labels lexicographically [i.e., $(n, i) \leqq(m, j)$ if $n<m$ or if $n=m$ and $i \leqq j]$. Using this labeling we form the future $Q_{0} P$-names ${ }^{33}$ of elements $x \in X$ and order them lexicographically using the lexicographical ordering of labels. Given $\bar{x} \in \bar{X}$, we index the particles in each element of $Q_{0} P$ according to this ordering. $\overline{\bar{P}}$ is the partition of $\bar{X}$ according to the number or particles in each of the elements of $P$, the element of $Q_{0} P$ containing, at $t=-1$, each of these indexed particles, and the indexed at $t=-1$ in their respective elements of

31 All partitions to which we henceforth refer are to be understood as measurable.

$32\left(Q_{0} P \vee T Q_{0} P\right) \cap R$ is finite since $T\left(R_{0}\right)$ is bounded.

33 If $P^{\prime}=\left\{P_{i}^{\prime}\right\}$ is any partition of a space $X$ with automorphism $T$, the future $P^{\prime}$-name of a point $x \in X$ is the sequence $\left(i_{1}, i_{2}, \ldots\right)$ when $T^{j} x \in P_{i_{j}}^{\prime}, j=1,2, \ldots$. 
$Q_{0} P$ of each of these $(t=0)$ indexed particles. One easily verifies that, like $P^{I}, \overline{\bar{P}}$ has finite entropy.

Using the remarks preceding Lemma 6.5 we may obtain that

$$
h(\overline{\mathscr{I}})=\sup _{P \text { finite }} h(\overline{\bar{P}}, \overline{\mathscr{I}}):
$$

we can easily construct an increasing sequence $\left\{P_{n}\right\}$ of finite partitions of $R_{0}$ for which $\bigvee_{n}\left(\overline{\bar{P}}_{n}\right)_{\bar{S}}=\varepsilon(\bmod 0)$. But

$$
h(\overline{\bar{P}}, \overline{\mathscr{I}})=H\left(\overline{\bar{P}} \| \overline{\bar{P}}_{\overline{\bar{I}}}\right)=\sum_{n=0}^{\infty}\left(e^{-\varrho} \varrho^{n} / n !\right) n \tilde{h}(P, T)=\varrho \tilde{h}(P, T),
$$

since the index information at $t=-1$ is determined by $\overline{\bar{P}}_{\bar{S}}$, particles coming from $R_{-n}, n>0$, at $t=0$, automatically having lower $t=-1$ indices than particles from $R_{0}$, which in turn have lower $t=-1$ indices than particles from $R_{m}, m>0$. Taking the supremum over $P$ finite leads to the desired result.

Proof of Lemma 6.7. We have that

$$
\begin{aligned}
h\left(\bar{T}_{R}\right) & =\sum_{n=0}^{\infty}\left(e^{-\mu(R)} \mu(R)^{n} / n !\right) h\left(T_{R \text { symm }}^{\times n}\right) \\
& =\sum_{n=0}^{\infty}\left(e^{-\mu(R)} \mu(R)^{n} / n !\right) h\left(T_{R}^{\times n}\right) \\
& =\sum_{n=0}^{\infty}\left(e^{-\mu(R)} \mu(R)^{n} / n !\right) n h\left(T_{R}\right) \\
& =n h\left(T_{R}\right) .
\end{aligned}
$$

The first equality follows from the fact that the entropy of a direct sum is the average of the entropies ${ }^{34}$. The second equality follows in a manner similar to the proof of Lemma 6.6.

$$
\left[h\left(P^{\times n}, T^{\times n}\right)=h\left(P_{\text {symm-indexed }}^{\times n}, T_{\text {symm }}^{\times n}\right),\right.
$$

where $T$ is an automorphism of a probability space $(X, \Sigma, \mu)$ and $P_{\text {symm-indexed }}^{\times n}$ is the partition of $X_{\text {symm }}^{n}$ according to membership in the elements of the partition $P$ of the (future $P$-name) indexed "particles".]

$$
\begin{aligned}
34(1 / n) H\left(\bigvee_{j=0}^{n-1} T^{j}(P \vee \gamma)\right)= & (1 / n) H\left(\left(\bigvee_{j=0}^{n-1} T^{j} P\right) \vee \gamma\right) \\
= & (1 / n) H(\gamma)+(1 / n) H\left(\bigvee_{j=0}^{n-1} T^{j} P \| \gamma\right) \\
& \underset{n \rightarrow \infty}{\longrightarrow} \sum_{k} \mu\left(\gamma_{k}\right) h\left(P \cap \gamma_{k}, T_{\gamma_{k}}\right),
\end{aligned}
$$

where $T$ is an automorphism of a probability space with (invariant) components $\gamma_{k}, \gamma=\left\{\gamma_{k}\right\}$ is the partition into components, $P \cap \gamma_{k}$ is the restriction of $P$ to $\gamma_{k}$, and $T_{\gamma_{k}}$ is the restriction of $T$ to $\gamma_{k}$. 
The third equality follows from the well known fact that the entropy of a direct product is the sum of the entropies [since

$$
\left.H\left(P_{1} \times P_{2}\right)=H\left(P_{1}\right)+H\left(P_{2}\right)\right] .
$$

We have thus shown that our expectations are realized for systems of periodic-bounded-type: the space-time entropy equals the time entropy per unit volume (in any rectangle $R$ ) which in turn equals the time entropy of a single particle moving in any rectangle times the average number of particles per unit volume.

We would like these results to be valid for systems such as the Lorentz gas, for which $T\left(R_{0}\right)$ is not bounded because $R_{0}$ contains points corresponding to particles with arbitrarily high velocity, but which is nonetheless of periodic- $\sigma$-bounded-type (see Section 2$)^{35}$. (The speed of a particle is a constant of the motion for the Lorentz gas.) Since systems of periodic- $\sigma$-bounded-type are in a strong sense limits of systems of periodic-bounded-type we should be able to "go to the limit" and obtain

Theorem 6.8. If $(\bar{X}, \bar{\Sigma}, \bar{\mu}, \bar{T})$ is of periodic- $\sigma$-bounded type, then

$$
h(\overline{\mathscr{I}})=h_{1}(\bar{T})=\mu\left(R_{0}\right) h\left(T_{R}\right)=(1 /\|R\|) h\left(\bar{T}_{R}\right) .
$$

Proof. Let $X=\bigcup_{n} X_{n}$ display $(\bar{X}, \bar{\Sigma}, \bar{\mu}, \bar{T})$ as a system of periodic- $\sigma$ bounded-type (see Section 2). Let $\Sigma\left(X_{n}\right)$ be the (invariant) sub- $\sigma$-algebra of $\Sigma$ corresponding to the measurable partition of $X$ into the set $X-X_{n}$ and the points of $X_{n}$. Let us denote the system $(X, \Sigma, \mu, T)$ by $\tau$, and let $\tau\left(X_{n}\right)$ be the factor of $\tau$ with respect to $\Sigma\left(X_{n}\right)^{36}$. Then $\bar{\tau}\left(X_{n}\right)$ is "essentially" of periodic-bounded-type, since $\tau\left(X_{n}\right)$ can be expressed as the direct sum

$$
\tau\left(X_{n}\right)=\tau^{\prime}\left(X_{n}\right) \oplus \mathbb{1}\left(X_{n}\right),
$$

where $\tau^{\prime}\left(X_{n}\right)$ is the restriction of $\tau$ to $X_{n}$ and $\mathbb{1}\left(X_{n}\right)$ is the system consisting of a single (invariant) "point" (of infinite measure) - the set $X-X_{n}{ }^{37}$. Furthermore,

$$
\bar{\tau}\left(X_{n}\right)=\bar{\tau}^{\prime}\left(X_{n}\right) \times \overline{\mathbb{1}}\left(X_{n}\right) .
$$

Now, since $\tau^{\prime}\left(X_{n}\right)$ is of periodic-bounded-type we can apply Theorem 6.7 to obtain

$$
\begin{aligned}
h\left(\overline{\mathscr{I}}^{\prime}\left(X_{n}\right)\right) & =h_{1}\left(\bar{T}^{\prime}\left(X_{n}\right)\right)=(1 /\|R\|) h\left(\bar{T}_{R}^{\prime}\left(X_{n}\right)\right) \\
& =\mu\left(X_{n} \cap R_{0}\right) h\left(T_{R}^{\prime}\left(X_{n}\right)\right) .
\end{aligned}
$$

35 For the proof of Theorem 6.7 the boundedness of $T\left(R_{0}\right)$ was used only to guarantee the finiteness of the entropy of $\left(T Q_{0}\right) \cap R_{0}$. Therefore the conclusion of Theorem 6.7 in fact applies to the Lorentz gas (Maxwellian velocity distribution); the following theorem indicates that for systems of periodic- $\sigma$-bounded-type no assumption concerning the entropy of $T Q_{0} \cap R_{0}$ or, in particular, the velocity distribution is necessary.

36 The factor of a system with respect to an invariant sub- $\sigma$-algebra $\Sigma^{\prime}$ is the system obtained by identifying points in the same element of $\hat{\Sigma}^{\prime}$.

37 We denote, as usual, the Poisson system built over $\tau$ by $\bar{\tau} \ldots$. 
We also have that

$$
\begin{aligned}
& h\left(\overline{\mathscr{I}}\left(X_{n}\right)\right)=h\left(\overline{\mathscr{I}}^{\prime}\left(X_{n}\right)\right) ; h\left(\bar{T}_{R}\left(X_{n}\right)\right)=h\left(\bar{T}_{R}^{\prime}\left(X_{n}\right)\right) ; \\
& h\left(T_{R}\left(X_{n}\right)\right)=\left(\mu\left(X_{n} \cap R_{0}\right) / \mu\left(R_{0}\right)\right) h\left(T_{R}^{\prime}\left(X_{n}\right)\right) .
\end{aligned}
$$

By virtue of the remarks immediately prior to Lemma 6.5, the desired result follows upon taking the limit $n \rightarrow \infty$ (using, for example, the definition of a measurable partition $[13,14]$ and a diagonalization argument).

Finally, we "calculate" the space-time entropy of the Lorentz gas. Let $\tau(\equiv(X, \Sigma, \mu, T))$ be the base system of the Lorentz gas, and let $\tau_{s}$ be the component of $\tau$ corresponding to a particle with speed $s . \tau$ is the direct integral of its components $\tau_{s}$. We have shown that the space-time entropy of $\tau$ is $\varrho h\left(T_{R_{0}}\right)$. Using the representation of the entropy of a direct integral as the integral of the entropies of the components [19] and the formula of Abramov (see Section 3g), we obtain that

$$
h\left(T_{R_{0}}\right)=\int_{0}^{\infty} h\left(T_{R_{0}, s}\right) \lambda(d s)=h\left(T_{R_{0}, 1}\right) \int_{0}^{\infty} s \lambda(d s)
$$

where $\lambda$ is the distribution on the speed of a particle induced by $\mu$. Thus

$$
h(\overline{\mathscr{I}})=\varrho\langle s\rangle_{\lambda} h\left(T_{R_{0}, 1}\right),
$$

consistent with the interpretation of it as a measure of the loss of information (due to "collisions") per unit volume per unit time.

\section{Concluding Remarks}

The results of the previous section, in addition to indicating that the space-time entropy of the systems we have considered has a natural interpretation, establish that the time entropy per unit volume is an invariant (see $[8,10]$ ) of our expanded framework, at least for the class of systems of the kind considered. We also have the desirable result that two such systems for which the "dissipation" per unit volume occurs at different rates cannot be isomorphic.

We would like these results to extend to general translation invariant equilibrium states of Hamiltonian systems (for which the infinite volume time evolution can be appropriately defined [20-22]). The concept of time entropy per unit volume could be defined by using, say, sequences of cubes with either reflecting of periodic boundary conditions [21]. We might then expect the space-time entropy of the equilibrium states of these systems to equal the time entropy per unit volume, so that the "local rate of dissipation" would be an invariant of a larger class of systems, including all systems of physical significance. 
We have found that a system (e.g., the ideal gas) may be Bernoulli under both space translation and time evolution separately without being even a space-time $K$-system, not to mention Bernoulli. We have not found any models of realistic systems which are space-time Bernoulli, though we can give a characterization of such systems which makes clearer what is involved. It is clear that if a system is Bernoulli under the space-time group, it is Bernoulli under space translation $(S)$ and possesses an $S$-invariant independent generator for $T$ (the time evolution). The converse is also true. Indeed, since factors of Bernoulli shifts are Bernoulli [7], any $S$-invariant independent generator for $T$ can be expressed in the form $\xi_{S}$, with $\xi, S \xi, S^{2} \xi, \ldots$ forming an independent sequence of partitions, so that $\xi$ is, in fact, an independent generator for $(S, T)$.

We give a simple example of a class of $(S, T) B$-systems: Let $(B, \tilde{T})$ be a Bernoulli scheme. (We are here dropping explicit reference to the measure.) Let $X=B^{\mathbb{Z}}$ (measure theoretic product) and $T=\tilde{T}^{\mathbb{Z}}$ and let $S$ act in the obvious way as a translation on $B^{\mathbb{Z}}$. It is clear that $\underset{i}{X} Q_{i}\left(Q_{i}=B\right.$ for $i \neq 0, Q_{0}=P$, an independent generator for $\tilde{T}$ ) is an independent generator for $(S, T)$.

We observe that though, e.g., the Lorentz gas clearly exhibits better thermodynamic behavior than, say, the ideal gas, a nonequilibrium (i.e., non-Maxwellian) velocity distribution for it does not approach, as the time approaches infinity, the appropriate Maxwellian distribution. This is, perhaps, not at all to be unexpected because velocities are not very "natural" within the framework of discrete symmetry (spatial for the Lorentz gas). A question of interest might be the behavior of the velocity distribution in systems with continuous symmetry $(\mathscr{I}=$ full space-time group) and strong (say, $K$ or Bernoulli) $\mathscr{I}$-ergodic properties. Generalized dynamical systems with continuous symmetry can be obtained in the obvious way from systems of interacting particles, which we would hope exhibit the appropriate behavior of the velocity distribution functions.

Finally, we note a connection between systems with completely positive entropy (in particular, $K$-systems) and the possibility of "finite approximation" of a dynamical system. A Hamiltonian system (say) whose time evolution has completely positive entropy does not admit of a finite approximation, in the sense that no coarse-grained (finite) observation-corresponding to a finite partition-performed at regular intervals (at times $t=-\tau,-2 \tau,-3 \tau, \ldots$ ) throughout the entire past can always determine with absolute certainty the outcome of the measurement at $t=0$, and conversely. It is therefore not surprising that the time evolution of an infinite system should form a $K$-system ( $\equiv$ completely positive entropy for single automorphism [14]), since it is not very 
plausible that a (realistic) infinite system should be finitely approximable. For a system with completely positive space-time entropy, however, something much stronger is true: it is not even locally finitely approximable, in the sense that no finite local observation, performed at regular intervals throughout all of space and the entire past, is sufficient to always determine the outcome of a present performance of that observation.

\section{Appendix I \\ Fock Space Representation of Induced Unitaries of a Poisson System ${ }^{38}$}

Let $(\bar{X}, \bar{\Sigma}, \bar{\mu})$ be the Poisson probability space built over $(X, \Sigma, \mu)$. Let us denote $L^{2}(\mu)$ by $\mathscr{H}$ and let us write $\mathscr{H}_{n}$ for $\mathscr{H}_{\text {symm }}^{\otimes n}, n=0,1,2,3, \ldots$ $\left(\mathscr{H}_{0}=\mathbb{C} \cdot \mathbb{1}\right)$. We identify $\mathscr{H}_{n}$ with the space of all symmetric square integrable functions on $\left(X^{n}, \Sigma^{\times n}, \mu^{\times n}\right)$. We will show in particular that $L^{2}(\bar{\mu})$ may be identified with the boson Fock space built over $\mathscr{H}\left(\equiv \bigoplus_{n=0}^{\infty} \mathscr{H}_{n}\right)$ in such a way that $U_{\bar{T}}$ is identified with $\bigoplus_{n=0}^{\infty} U_{T}^{\otimes n}[$ for all automorphisms $T$ of $(X, \Sigma, \mu)]$, which follows from the

Theorem. There exists a sequence of unitary mappings

$$
\hat{\Sigma}_{n}: \mathscr{H}_{n} \rightarrow L^{2}(\bar{\mu}), \quad n=0,1,2, \ldots{ }^{39}
$$

such that

1. $\hat{\Sigma}_{n}\left(U_{T}^{\otimes n} f\right)=U_{\bar{T}} \hat{\Sigma}_{n} f$ for all $f \in \mathscr{H}_{n}, n \geqq 1$ (and all automorphisms $T$ ).

2. $\hat{\Sigma}_{n} \mathscr{H}_{n}$ is orthogonal to $\hat{\Sigma}_{m} \mathscr{H}_{m}$ for all $m \neq n$.

3. $L^{2}(\bar{\mu})=\bigoplus_{m=0}^{\infty} \hat{\Sigma}_{m} \mathscr{H}_{m}$.

4. If $\Lambda$ is a subset of $X$ with finite measure, and if $f\left(x_{1}, \ldots, x_{n}\right) \in \mathscr{H}_{n}$ is zero a.e. outside of $\Lambda$, then $\hat{\Sigma}_{n} f$ is measurable in $\Lambda$.

Proof. We proceed as follows: We first assume $\mu(X)=\infty$; we prove analogues of 1 . and 2 . for dense subsets of the $\mathscr{H}_{n}$ and extend to all the $\mathscr{H}_{n}$, obtaining 3 . and 4 . in the process. We then use 4 . to remove the restriction $\mu(X)=\infty$.

Let $\tilde{\mathscr{H}}$ denote the set of all square integrable functions on $(X, \Sigma, \mu)$ with support in some $\Lambda$ with $\mu(\Lambda)<\infty$ [i.e., $f(x)=0$ a.e. outside of $\Lambda$ ] and with $\int f d \mu=0$. $\tilde{\mathscr{H}}$ is dense in $\mathscr{H}$ in view of our assumption that $\mu(X)=\infty$. Let $\tilde{\mathscr{H}}_{n}$ denote the $n$-fold algebraic tensor product of $\tilde{\mathscr{H}}$

\footnotetext{
${ }^{38}$ The material of this appendix is due to Oscar Lanford.

${ }^{39} \hat{\Sigma}_{0}: \mathscr{H}_{0} \rightarrow \mathbb{C} \cdot \mathbb{1} \subset L^{2}(\bar{\mu})$ by inclusion mapping.
} 
with itself regarded as a linear subset of $\mathscr{H}_{n}$. Now $\tilde{\mathscr{H}}_{n}$ is dense in $\mathscr{H}_{n}$ and for $f_{n} \in \tilde{\mathscr{H}}_{n}$

$$
\int \mu\left(d x_{i}\right) f_{n}\left(x_{1}, \ldots, x_{n}\right) \equiv 0
$$

for all $i$. Define $\Sigma_{n}$ for $f \in \mathscr{H}_{n}$ with support in some $\Lambda$ by

$$
\left(\Sigma_{n} f\right)\left(\left\{x_{i}\right\}\right)=\sqrt{n !} \sum_{i_{1}<\cdots<i_{n}} f\left(x_{i_{1}}, \ldots, x_{i_{n}}\right)
$$

(a function on $\bar{X}$ ). A straightforward computation indicates that for each $n, \Sigma_{n}$ defines a unitary mapping of $\tilde{\mathscr{H}}_{n}$ into $L^{2}(\bar{\mu})$ such that $\Sigma_{n} \tilde{\mathscr{H}}_{n}$ is orthogonal to the constants and to $\Sigma_{m} \tilde{\mathscr{H}}_{m}$ for $m \neq n$. We define $\hat{\Sigma}_{n}$ through extension by continuity, immediately obtaining 1 . and 2 . of the theorem. 4. is valid for $f \in \tilde{\mathscr{H}}_{n}$; to establish it for all of $\mathscr{H}_{n}$ we compute $\hat{\Sigma}_{n} f$ for $f \in \mathscr{H}_{n}$ vanishing outside of $\Lambda$. If, for example, $f \in \mathscr{H}$, we find a sequence $f^{(m)} \in \tilde{\mathscr{H}}$ converging to $f$ in $L^{2}(\mu)$; then

$$
\hat{\Sigma}_{1} f=\lim _{m \rightarrow \infty} \Sigma_{1} f^{(m)} .
$$

We may construct the $f^{(m)}$ by forming a sequence $M_{m}$ of subsets of $X$ with $\mu\left(M_{m}\right) \rightarrow \infty\left(\mu\left(M_{m}\right)<\infty\right)$ and put

$$
f^{(m)}(x)=f(x)-\left(\varphi_{M_{m}}(x) / \mu\left(M_{m}\right)\right) \int f d \mu^{40} .
$$

The latter term clearly converges to zero in $L^{2}(\mu)$. Also,

$$
\Sigma_{1} f^{(m)}=\Sigma_{1} f-\left(\int f d \mu\right) N_{M_{m}} / \mu\left(M_{m}\right) .
$$

Since $N_{M_{m}} / \mu\left(M_{m}\right)$ converges to the constant function $\mathbb{1}\left[\right.$ in $\left.L^{2}(\bar{\mu})\right]$, we have

$$
\hat{\Sigma}_{1} f=\Sigma_{1} f-\int f d \mu .
$$

Proceeding in a similar manner, we may express $\hat{\Sigma}_{n} f_{n}$, for $f_{n} \in \mathscr{H}_{n}$ vanishing outside some $\Lambda$, as a linear combination of the form

where

$$
\hat{\Sigma}_{n} f_{n}=\Sigma_{n} f_{n}+\sum_{j=0}^{n-1} c_{j} \Sigma_{j} f_{j}\left(\Sigma_{0} \equiv \hat{\Sigma}_{0}\right)
$$

$$
f_{j}\left(x_{1}, \ldots, x_{j}\right)=\int \mu\left(d x_{j+1}\right) \ldots \mu\left(d x_{n}\right) f_{n}\left(x_{1}, \ldots, x_{n}\right),
$$

displaying $\hat{\Sigma}_{n} f_{n}$ as a function measurable in $\Lambda$. We see also that the finite linear span of functions of the form $\hat{\Sigma}_{n} f_{n}$ contains all functions of the form $\Sigma_{n} f_{n}$. Since functions of the form

$$
\exp \left(i \theta N_{A}\right)=\sum_{n=0}^{\infty}\left(i \theta N_{A}\right)^{n} / n !
$$

are in the closed linear span of functions of the form $\Sigma_{n} f_{n}$, we have established 3.

\footnotetext{
${ }^{40} \varphi_{M}$ is the characteristic function of $M \subset X$.
} 
Finally, if $\mu(X)$ is finite, we replace $(X, \mu)$ by $\left(X \cup X^{\prime}, \mu \oplus \mu^{\prime}\right)$, where $\left(X^{\prime}, \mu^{\prime}\right)$ is an infinite measure space. This replaces $(\bar{X}, \bar{\mu})$ by $\left(\bar{X} \times \bar{X}^{\prime}\right.$, $\left.\bar{\mu} \times \bar{\mu}^{\prime}\right)$. Since 4 . implies that if $f \in \mathscr{H}_{n}(X), \hat{\Sigma}_{n} f \in L^{2}(\bar{\mu})$, the proof is complete.

\section{Appendix II}

\section{Proof of Lemma 5.5}

The finiteness of $H\left(P^{I}\right)$ follows from elementary estimates, using the following:

a) For measurable partitions $\alpha, \beta$, and $\delta$,

$$
\begin{aligned}
H(\alpha \vee \beta \| \delta) & =H(\alpha \| \delta)+H(\beta \| \alpha \vee \delta) \\
& \leqq H(\alpha \| \delta)+H(\beta \| \delta) \quad(\text { see }[14]) .
\end{aligned}
$$

b) All moments of a Poisson distribution are finite.

c) All moments of a Gaussian distribution are finite.

d) For $\sum_{i=1}^{\infty} p_{i}=1$ and $p_{j} \geqq 0$ for all $j$,

$$
\sum_{n=1}^{\infty} p_{n} \log n \leqq \log \left(\sum_{n=1}^{\infty} n p_{n}\right)=\log \langle n\rangle,
$$

since $\log t$ is concave in $(0, \infty)$.

e) $H(P) \leqq \log k$ if $P$ has no more than $k$ atoms.

We estimate $H\left(P^{I}\right)$ by writing

$$
P^{I}=P_{1} \vee P_{2} \vee P^{I},
$$

where $P_{1}$ is the partition of $\bar{X}^{I}$ according to the number of particles in $[0,1) \times \mathbb{R}$, and $P_{2}$ is the refinement of $P_{1}$ according to the cell membership at $t=1$ of the indexed particles in $[0,1)$. We then have that

Now

$$
H\left(P^{I}\right)=H\left(P_{1}\right)+H\left(P_{2} \| P_{1}\right)+H\left(P^{I} \| P_{1} \vee P_{2}\right) .
$$

$$
H\left(P_{1}\right)=\tilde{H}(\varrho) \equiv-\sum_{n=0}^{\infty}\left(e^{-\varrho} \varrho^{n} / n !\right) \log \left(e^{-\varrho} \varrho^{n} / n !\right),
$$

where $\tilde{H}(0)=0$ and $\tilde{H}(t)$ is continuous for $t \in[0, \infty) . \quad\left(\varrho=\mu^{I}\left(R_{0}\right)\right.$ $=\mu^{I}([0,1) \times \mathbb{R}$.) We also have that,

$$
\begin{aligned}
H\left(P_{2} \| P_{1}\right) & =\sum_{n=0}^{\infty}\left(e^{-\varrho} \varrho^{n} / n !\right) H\left(P_{2} \cap \alpha_{n}\right) \\
& \leqq \sum_{n=0}^{\infty}\left(e^{-\varrho} \varrho^{n} / n !\right) n C_{\beta}<\infty
\end{aligned}
$$


where $\alpha_{n}=\left\{\bar{x} \in \bar{X}^{I} \mid N_{[0,1) \times \mathbb{R}}(\bar{x})=n\right\}$ and $C_{\beta}$ is a uniform (in $n$ and $i$ ) bound on the entropy of the partition of $\alpha_{n}$ (normalized to unit total measure) according to the cell membership at $t=1$ of the particle with index $i$ in $[0,1)^{41}$.

For $\gamma$ an atom of $P_{1} \vee P_{2}$, let $P_{\gamma, j}$ be the partition of $\gamma$ according to the index at $t=1$ of the particle with index $j$ in $[0,1)$. We have that

$$
\begin{aligned}
H\left(P^{I} \| P_{1} \vee P_{2}\right) & =\sum_{\gamma \in P_{1} \vee P_{2}} \bar{\mu}^{I}(\gamma) H\left(P^{I} \cap \gamma\right) \\
& \leqq \sum_{\gamma \in P_{1} \vee P_{2}} \bar{\mu}^{I}(\gamma) \sum_{j=1}^{n(\gamma)} H\left(P_{\gamma, j}\right)
\end{aligned}
$$

where $n(\gamma)=$ the value of $N_{[0,1) \times \mathbb{R}}$ characteristic of $\gamma$. Let $\tilde{P}_{\gamma, j}$ be the partition of $\gamma$ according to $N_{j}$, the number of particles at time $t=1$ in the cell containing the particle which at $t=0$ has index $j$ in $[0,1)$. Then

$$
H\left(P_{\gamma, j}\right) \leqq H\left(\tilde{P}_{\gamma, j} \vee P_{\gamma, j}\right)=H\left(\tilde{P}_{\gamma, j}\right)+H\left(P_{\gamma, j} \| \tilde{P}_{\gamma, j}\right) .
$$

Now, using e) and d), we obtain

$$
\begin{aligned}
H\left(P_{\gamma, j} \| \tilde{P}_{\gamma, j}\right) & \leqq \sum_{k=1}^{\infty} \tilde{p}_{k} \log k \leqq \log \sum_{k} k \tilde{p}_{k} \\
& \leqq \log (\varrho+n(\gamma)),
\end{aligned}
$$

where $\tilde{p}_{k}$ is the probability, given $\gamma$, that $N_{j}=k$. Also, $\tilde{P}_{\gamma, j}$ is the partition of $\gamma$ according to the value of $N_{A_{\gamma, j}}$, with

$$
A_{\gamma, j}=\left(T^{I}\right)^{-1}\left(\left[n_{j}(\gamma), n_{j}(\gamma)+1\right) \times \mathbb{R}\right)-[0,1) \times \mathbb{R}
$$

where $n_{j}(\gamma)$ is the left coordinate of the cell containing at $t=1$ the particle with index $j$ in $[0,1)$.

Thus $H\left(\tilde{P}_{\gamma, j}\right)=\tilde{H}\left(\mu^{I}\left(A_{\gamma, j}\right)\right)$, with $\mu_{\tilde{H}}^{I}\left(A_{\gamma, j}\right)<\varrho$, so it appears that $\tilde{H}\left(\mu^{I}\left(A_{\gamma, j}\right)\right) \leqq \tilde{H}(\varrho)$. In any case, since $\tilde{H}(t)$ is continuous for $t \in[0, \infty)$, we have $\tilde{H}\left(\mu^{I}\left(A_{\gamma, j}\right)\right) \leqq \tilde{H}(\tilde{\varrho})$ for some $\varrho \in(0, \varrho]$ not depending upon $\gamma$ and $j$.

41 In the general situation (see Footnote 35 ) we do not obtain a uniform bound; instead we reason as follows: Let $P=\left\{P_{i}\right\}$ and $\overline{\bar{P}}$ be as in the proof of Lemma 6.6. Since we may write $\overline{\bar{P}}=\overline{\bar{P}}_{1} \vee \cdots \vee \overline{\bar{P}}_{m}$, where $\overline{\bar{P}}_{i}$ is defined in the obvious manner, we may, by virtue of a), assume that $P$ is trivial. Let $Q=T Q_{0} \cap R_{0}$ and let $Q^{(i)}$ be the partition of $\left(R_{0}^{n},\left(\Sigma \cap R_{0}\right)^{\times n}\right.$, $\left.\left(\mu_{R_{0}} / \mu\left(R_{0}\right)\right)^{\times n}\right)$ according to the "position" of the $i$ th particle relative to $Q$. Let $\#_{i}$ be the index of the $i$ th particle (see proof of Lemma 6.6). We also denote by $\#_{i}$ the partition of $R_{0}^{n}$ determined by $\#{ }_{i}$. We have that $H(Q)<\infty$ and must show that $H\left(\left(Q^{(1)} \vee \cdots \vee Q^{(n)}\right)\right.$ $\left.\cap\left\{\#_{1}=1, \ldots, \#_{n}=n\right\}\right)<\infty$. But

$$
\begin{aligned}
& H\left(\bigvee_{i=1} Q^{(i)} \cap\left\{\#_{1}=1, \ldots, \#_{n}=n\right\}\right) \leqq \sum_{i=1}^{n} H\left(Q^{(i)} \cap\left\{\#_{i}=i\right\}\right) \\
& \quad=\sum_{i=1}^{n} H\left(Q^{(1)} \cap\left\{\#_{1}=i\right\}\right)=n H\left(Q^{(1)} \| \#_{1}\right) \leqq n H\left(Q^{(1)}\right)=n H(Q)<\infty .
\end{aligned}
$$


Thus,

$$
\begin{aligned}
H\left(P^{I} \| P_{1} \vee P_{2}\right) & \leqq \sum_{n=1}^{\infty} n \bar{\mu}^{I}\left(\alpha_{n}\right)(\tilde{H}(\varrho)+\log (n+p)) \\
& =\sum_{n=1}^{\infty}\left(e^{-\varrho} \varrho^{n} / n !\right)(n \tilde{H}(\varrho)+n \log (n+\varrho))<\infty,
\end{aligned}
$$

completing the proof.

Acknowledgement. I would like to thank Michael Aizenman, Oscar E.Lanford III, Joel L. Lebowitz, and Oliver Penrose for very valuable discussions and helpful suggestions. I also wish to thank the Institute for Advanced Study for the hospitality and financial support extended to me.

\section{References}

1. Goldstein, S., Lebowitz, J. L.: Commun. math. Phys. 37, 1-18 (1974)

2. Aizenmann, M., Goldstein, S., Lebowitz, J.L.: Ergodic properties of a one-dimensional system of hard rods with an infinite number of degrees of freedom. (To appear)

3. Lanford III,O.E., Lebowitz, J.L.: Ergodic properties of an infinite harmonic crystal. (To appear)

4. Volkovysskii, K. L., Sinai, Y. G.: Funkts. Analiz 5, No. 3, 19 (1971)

5. Sinai, Y.G.: Funkts. Analiz 6, No. 1, 41 (1972)

6. Gallavotti, G., Ornstein, D. S.: Billiards and Bernoulli schemes. Commun. math. Phys. (in press) (1974)

7. Ornstein,D.S.: Ergodic theory, randomness, and dynamical systems. New Haven: Yale Univ. Press 1974

8. Billingsley,P.: Ergodic theory and information. New York: Wiley 1965

9. Ruelle,D.: Statistical mechanics. Rigorous results. New York: Benjamin 1967

10. Arnold, V.I., Avez, A.: Ergodic problems of classical mechanics. New York: Benjamin 1968

11. Conze, J.P.: Z. Wahrscheinlichkeitstheorie verw. Geb. 25, 11-30 (1972)

12. Katznelson, Y., Weiss, B.: Israel J. Math. 12, 161 (1972)

13. Rohlin, V.A.: Am. Math. Soc. Transl. (1) 10, 1-54 (1962)

14. Parry, W.: Entropy and generators in ergodic theory. New York: Benjamin 1969

15. Halmos, P. R.: Measure theory. New York: Van Nostrand 1950

16. Goldstein, S., Lanford III, O.E.: Occupation number measures and the uniqueness of the state in classical statistical mechanics. (To appear)

17. Shields, P.: The theory of Bernoulli shifts. University of Chicago Press 1973

18. Sinai, Y.G.: Russ. Math. Surveys 25, 137 (1970)

19. Jacobs, K.: Lecture notes on ergodic theory. Aarhus: 1962/63

20. Lanford III, O.E.: Commun. math. Phys. 9, 176 (1968)

21. Lanford III, O.E.: Commun. math. Phys. 11, 257 (1969)

22. Lanford III, O.E.: (To appear)

Communicated by J. L. Lebowitz

Sheldon Goldstein

Institute for Advanced Study

Princeton, N.J. 08540, USA 
